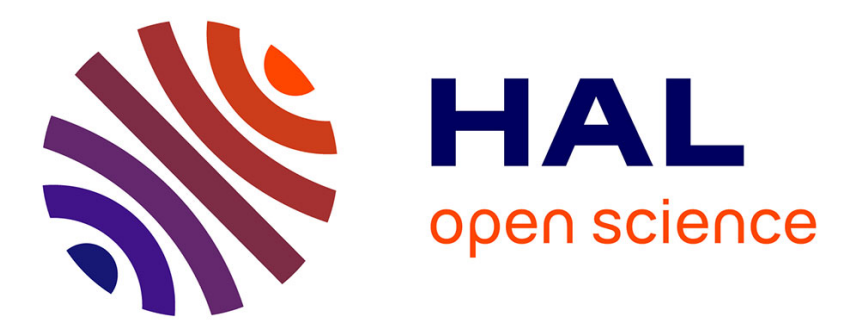

\title{
Optical properties of deposit models for paints: full-fields FFT computations and representative volume element
}

Dario F. Azzimonti, François Willot, Dominique Jeulin

\section{- To cite this version:}

Dario F. Azzimonti, François Willot, Dominique Jeulin. Optical properties of deposit models for paints: full-fields FFT computations and representative volume element. Journal of Modern Optics, 2013, 60 (7), pp.519-528. 10.1080/09500340.2013.793778 . hal-00836118

HAL Id: hal-00836118

https://hal-mines-paristech.archives-ouvertes.fr/hal-00836118

Submitted on 19 Dec 2014

HAL is a multi-disciplinary open access archive for the deposit and dissemination of scientific research documents, whether they are published or not. The documents may come from teaching and research institutions in France or abroad, or from public or private research centers.
L'archive ouverte pluridisciplinaire $\mathbf{H A L}$, est destinée au dépôt et à la diffusion de documents scientifiques de niveau recherche, publiés ou non, émanant des établissements d'enseignement et de recherche français ou étrangers, des laboratoires publics ou privés. 


\title{
Optical properties of deposit models for paints: full-fields FFT computations and representative volume element
}

\author{
D. Azzimonti ${ }^{a}$, F. Willot ${ }^{a *}$ and D. Jeulin ${ }^{a}$ \\ ${ }^{a}$ MINES ParisTech, PSL Research University, \\ Centre de Morphologie Mathématique, \\ 35, rue Saint Honoré, 77305 Fontainebleau CEDEX, France.
}

\begin{abstract}
A 3D model of microstructure containing spherical and rhombi-shaped inclusions "falling" along a deposit direction is used to simulate the distribution of nanoscale color pigments in paints. The microstructures anisotropy and length scales, characterized by their covariance functions and representative volume element, follow that of transerseley isotropic or orthotropic media. Full-field computations by means of the fast Fourier method are undertaken to compute the local and effective permittivity function of the mixture, as a function of the wavelength in the visible spectrum. Transverse isotropy is numerically recovered for the effective permittivity of the deposit model of spheres. Furthermore, in the complex plane, the transverse and parallel components of the effective permittivity tensor are very close to the frontiers of the Hashin-Shtrikman's domain, at all frequencies (or color) of the incident wave. The representative volume element for the optical properties of paint deposit models are studied. At fixed accuracy, it is much larger for the imaginary part of the permittivity than for the real part, an effect of the strong variations of the electric displacement field, exhibiting hot-spots, a feature previously described in the context of conductivity.
\end{abstract}

Keywords Homogenization; Optical properties; Representative volume element; Deposit models; Random media; FFT method

\section{Introduction}

The purpose of this paper is to propose a quantitative approach to model the nanostructure of a paint and to predict its optical properties. This work is part of the ANR research

\footnotetext{
${ }^{*}$ Corresponding author. Email: francois.willot@ensmp.fr. To cite this article: D. Azzimonti, F. Willot and D. Jeulin, Journal of Modern Optics 60:7 519-528 (2013).
} 
project LIMA (www.lima-project.org), aiming to design computer-aided formulations of paints.

The optical properties of a paint are the result of the interaction between light and its components. The overall optical properties of a coating can be described by the optical indices $n$ derived from the wavelength depending dielectric permittivity $\varepsilon$ of the medium by means of the relation $n=\sqrt{\varepsilon}$ [13]. In the case of a paint, the coating is a mixture of different components with different permittivity, and its optical properties are ruled by the apparent effective permittivity of the mixture. Automotive paint, like other types of paint, is a complex compound made of many elements. The three main component of paint are binder, pigments and solvent. The binder, usually made of natural or synthetic resins, is the film-forming component for paint and its main component. The pigment (or filler) are particles that generate the color of paint. It is usually made of metals oxides like titanium $\left(\mathrm{TiO}_{2}\right)$, iron(III) $\left(\mathrm{Fe}_{2} \mathrm{O}_{3}\right)$ or alumina $\left(\mathrm{Al}_{2} \mathrm{O}_{3}\right)$. The chemical composition of the pigments defines the color of paint and the shape of the droplets of pigments. Finally, the solvent is the diluent used to dissolve the binder and adjust the viscosity of paint. Its contributions to the overall dielectric properties of paint are negligible, and therefore not considered in this work. Accordingly, paint is modeled as a two phases composite with a binder matrix and inclusions of pigments with different local permittivity. The present model is not limited to two phases structures as there could be microstructures with different pigments types.

Nanoparticles of pigments like hematite can show a broad variety of different shapes, depending on their fabrication conditions: pseudocubic particles [22], porous nanorods [29], nano-ellipsoid pigments [28], hollow spindle particles [15], nanorings [4]. Hierarchical structures can be obtained, such as flower-like aggregates [5] or core/shell particles [14]. In [16], optical properties of ellipsoidal hematite nanoparticles are measured on specimens. This variety of shapes for particles of a given pigment is expected to provide a wide range of optical properties of a paint. In addition to the shape of individual particles, their distribution in space (such as alignments, clusters, or isotropic arrangement) is expected to change the optical properties of a mix, and therefore the final aspect of a coating.

The color of paint is induced by the microstructure and the contrast between a matrix with real permittivity and oxide or metallic inclusions with complex permittivity. The imaginary part of the complex permittivity, even if small compared to the real part, is the main contribution to the color of a pigment as it accounts for the dissipative part of the tensor. When a light ray hits the microstructure of paint it is reflected completely by the resins, of real permittivity, and reflected with dissipation by the pigments. This dissipation allows only certain frequencies to be reflected therefore giving a particular color to the paint.

Examples of paint microstructures with inclusions similar to the ones used in the automotive industry may be found in $[23,2]$. The typical radius for a spherical pigment of $\mathrm{Al}_{2} \mathrm{O}_{3}$ is between $1 \mathrm{~nm}$ and $5 \mathrm{~nm}$. As for $\mathrm{TiO}_{2}$ we have that the diameter of the polyhedral crystals varies between 10 and $60 \mathrm{~nm}$. Figure 1 shows the shape of $\mathrm{Fe}_{2} \mathrm{O}_{3}$ droplets. The image shows rhomboidal shape pigments with a greater diagonal between 40 and $70 \mathrm{~nm}$. For such a range of sizes, well below the wavelength of the natural light, the quasistatic approximation rules out the apparent permittivity and therefore it can be estimated using 
the equations of electrostatics, as, made in section 3 below.

This paper is organized as follow: deposit models are introduced in section 1, optical properties and numerical computations are introduced in 3. Results for the effective permittivity tensors are discussed in sections 4 and 5. Finally computations of the representative volume element are given in section 6 .

\section{The Deposit Model}

Microstructure models suitable for paints should take into account the anisotropy induced by the application of droplets [2]. During the paint forming process, the pigments undergo the influence of a force, be it gravity or the force applied by a spray bell, which is statistically oriented along the same direction. This unidirectional force is not taken into account in traditional hard-core models of non overlapping particles, which are purely isotropic. On the contrary, in the hypothesis where the droplets are isotropic, this unidirectional force induces transversely isotropic microstructures. Deposit models, as described below, are instead more appropriate for the modeling of paints.

\section{$2.13 \mathrm{D}$ deposit models}

The "deposit model" considered in this work is derived from the theory of random sets originally introduced by G. Matheron [17]. Deposit models are RACS ("random closed sets") with a privileged direction called the deposit direction, and make use of a convex RACS called the elementary grain. Its definition is briefly recalled below.

Hereafter, we set $n=3$ and use the coordinate system $\left(\mathbf{e}_{x}, \mathbf{e}_{y}, \mathbf{e}_{z}\right)$ where $\mathbf{v}=-\mathbf{e}_{z}$ is the deposit direction. Additionally the domain $E$ containing the microstructure is chosen as a cube $Q \subset \mathbb{R}^{3}$ of size $L$. The highest $z$-coordinate $\bar{z}=\sup _{Q} z$, defines the top $z$ cut in $Q$ as $\pi=\{(x, y, \bar{z}) \in Q\}$. Inclusions "fall" in the direction $\mathbf{v}$ from the top $z$-plane until it touches either the microstructure's floor or another inclusion. Grains are inserted from an elementary grain $A^{\prime}$ and a $2 \mathrm{D}$ Poisson process $\Phi_{\bar{z}}=\left(x_{i}, y_{i}\right)_{i \in I}$ in the square $\pi \cap Q$, i.e. the grain $A^{\prime}$ is moved in the direction $\mathbf{v}$ until it hits either the bottom of the cube $z_{m}=\inf _{Q} z$ or another already inserted grain. This is repeated until it is no longer possible to place an elementary grain in the microstructure. More precisely, the "stop condition" is as follows: the algorithm stops when $n_{1}$ inclusions could not be inserted in the microstructure; moreover it tries to insert each inclusion $n_{2}$ times (from $n_{2}$ random points). Slightly higher volume fractions are achieved by choosing $n_{1}, n_{2}>1$; in the simulations $n_{1} \approx 600$ and $n_{2}=5$ have been chosen.

It is noted that even if the grains are isotropic, the deposit model is expected to be anisotropic along the direction $\mathbf{v}$. For instance, if the elementary grains are spheres, the model is transversely isotropic along $\mathbf{v}$. In the other directions, transverse to $\mathbf{v}$, the microstructure should have the same anisotropy as the grains. Moreover the model is by construction stationary (invariant over translations), and ergodic, which means that the spatial averages of the properties of the model approximate the expected value over realizations. These properties will be useful for determination of the representative volume element in Section 6. One common drawback of deposit models is the limited range 
of attainable volume fractions, which are often low. The deposit models method will accordingly be enhanced in order to achieve higher volume fractions.

\subsection{Microstructure simulation}

The deposit model is modified so as to introduce periodicity in directions transverse to $\mathbf{v}$, i.e. $x$ and $y$. This generalization is straightforward. The periodicity has been introduced for two main reasons. First, the microstructure should be seen as a small representative volume of the microstructure for which boundary effects are undesirable, and periodicity reduce them. Second, the numerical FFT method used to compute effective properties assume periodic boundary conditions. The latter are more consistent with periodic microstructures.

The most common pigment shapes observed in literature for automotive paint are spherical droplets and polygonal droplets, as discussed in Section 1, although more fancy shapes such as cuboids, diamonds, roses, cigars have been manufactured (see e.g. [23] for some examples). Due to the variety of pigments used in the automotive industry, the shapes considered here are not comprehensive. The grain shapes considered in this work consist of (i) spheres with identical radius $r=(1 / 25) L$; (ii) spheres with radius chosen as a random uniform variable between $(1 / 30) L$ and $(1 / 60) L$ (iii) rhombus-shaped inclusions. The latter are parametrized as $3 \mathrm{D}$ structure with two greater and minor diagonals $d_{1}=$ $(1 / 10) L$ and $d_{2}=0.7 d_{1}$ and a width $h=(7 / 100) L$, consistently with images in Figure 1 . Finally, each grain is either (iii-a) left as is; (iii-b) randomly rotated in the $(x, y)$ plane; (iiic) randomly rotated in the $(x, y)$ plane and in the $(y, z)$ plane. Obtained volume fractions vary between $12 \%$ and $23 \%$ and is dependent on the size of the inclusions with respect to $L$ and of the rotations.

Figures 2 shows examples of microstructures (i), (iii-b) and (iii-c) with spheres and rhombi inclusions. Rotation angles used to generate microstructure (iii-c) are uniform random variables in $[0, \pi / 2]$. The resulting volume fraction is about $18 \%$ for the microstructures (i) and (iii-b) and $10 \%$ for microstructure (iii-c). Resolutions of $600^{3}$ to $1200^{3}$ voxels (namely $0.6 \mu \mathrm{m}^{3}$ to $1.2 \mu \mathrm{m}^{3}$ ) were considered; for microstructure (i), these contain about 690 and 5,500 inclusions, respectively. It is noted that the algorithm for generating the grains and the deposit generation are separated. To minimize discretization effects, rotations are handled analytically (i.e. in a vectorial way).

\subsection{Morphological properties of simulations}

The covariance function $C(\mathbf{h})$ is useful to quantify the typical lengths and privileged directions of deposit microstructure. It is defined for the set $A$, as the probability $P$ for two points to be in $A$ :

$$
C(\mathbf{h})=P\{x \in A, x+\mathbf{h} \in \mathbf{A}\}
$$

The covariance function $C\left(h \mathbf{e}_{x, y, z}\right)$ in the three directions $x, y$ and $z$ is shown in Figure (3) for a deposit model made of spheres of equal radius (10 voxels). The function is equal to $C_{Q}(0)=0.16$, the spheres volume fraction, when $\mathbf{h}=0$. The strong anisotropy of the deposit model results in highly different covariance functions in the $x, y$ and $z$ direction. 
As expected however, due to the symmetries of the spheres model, covariances in the $x$ and $y$ directions are almost the same. One can observe that the covariance reaches a local minimum for a distance $|\mathbf{h}|$ close to the diameter of the spheres. This is the property of a hard-core process, and is a known consequence of the repulsion distance between centers of spheres resulting from the non-overlapping condition. The covariance shows two scales in the deposit direction, the larger scale obtained when the covariance reaches its asymptotic value $C(\mathbf{0})^{2}$ (for a range $A_{z} \approx 0.14=168$ voxels) corresponds to the typical size of aggregates (8-9 spheres) generated by the deposition.

\section{$3 \quad$ Local and effective optical properties}

The physical behavior of materials is often given in terms of macroscopic constitutive laws; they describe the responses of the system to some given solicitation. The local constitutive equations assume that the material is a continuous medium, and, in many cases of interest, offer a very good approximation of the properties when atomistics effects are negligible. For a brief overview of homogenization and effective properties in the context of electric or optical properties, see $[8,11]$.

When the typical size of the microstructure heterogeneities are much smaller than the light wavelengths, typically in the range $300-500 \mathrm{~nm}$ for the visible spectrum, electrostatics rule the calculation of the apparent dielectric permittivity. This is in particular valid for nanometric pigments. The system is accordingly governed by the quasi-static Maxwell equations:

$$
\nabla \cdot \mathbf{D}=0, \quad \nabla \times \mathbf{E}=0,
$$

where the vector fields $\mathbf{D}$ and $\mathbf{E}$ are the electric displacement and electric field, respectively, assuming the charge density is negligible. Moreover the electric field and the electric displacement are related by the constitutive equation $\mathbf{D}=\varepsilon \mathbf{E}$ where $\varepsilon$ is the permittivity of the local phase and is heterogeneous. It is noted that the condition $\nabla \times \mathbf{E}=0$ implies $\mathbf{E}=-\operatorname{grad} \Phi$ where $\Phi$ is the potential field associated to $\mathbf{E}$.

The above equations are completed with periodic boundary conditions on the unit square $Q$

$$
\mathbf{E}, \mathbf{D} \text {, periodic, }\langle\mathbf{E}\rangle_{Q}=\mathbf{E}_{0} \text {. }
$$

By linearity, the overall displacement reads $\langle\mathbf{D}\rangle_{Q}=\varepsilon^{*} \cdot \mathbf{E}_{0}$ where the (second-order) symmetric tensor $\varepsilon^{*}$ is the microstructure apparent permittivity. The tensor components are computed by means of the average field $\langle\mathbf{D}\rangle_{Q}$ for $\mathbf{E}_{0}=\mathbf{e}_{x}, \mathbf{e}_{y}$ and $\mathbf{e}_{z}$. Components of $\varepsilon^{*}$ should be averaged over different realizations; assuming ergodicity, one microstructure gives an approximation of the effective permittivity of the considered model if it is large enough. In particular, the transverse isotropic nature of the permittivity tensor is only recovered after averaging or if finite size effects are negligible. In this case, the effective tensor is defined by its diagonal components $\varepsilon_{x x}^{*} \approx \varepsilon_{y y}^{*}$ and $\varepsilon_{z z}^{*}$, whereas $\varepsilon_{x y}^{*}$, $\varepsilon_{x z}^{*}$ and $\varepsilon_{y z}^{*}$ are of several orders smaller.

Hereafter, the matrix is made of glass and the inclusions are $\mathrm{TiO}_{2}$ droplets. For the local permittivity of the two components values as measured in [24], are used. They are 
shown in Figure 4, where the permittivity values for the glass and $T_{i} O_{2}$ are represented as functions of the wavelength of incident wave.

Full-fields computations are undertaken using the Fast Fourier FFT algorithm of Eyre and Milton [3] and the software Morph'hom, developed at the Centre de Morphologie Mathématique. It is especially efficient for computing the response of large-sizes systems [26], and to deal with local singularities in anisotropic or nonlinear composites [27,6]. Figure 5 is an example of the fields in the microstructure. The image represents the real and imaginary part of the dielectric displacement $\mathbf{D}$ along the $x$ axis when a macroscopic electric field $\mathbf{E}_{0}=\mathbf{e}_{x}$ is applied. The field is stronger where the colors are brighter. As expected, this image shows higher average displacement in the inclusions than in the embedding matrix. The applied average field, oriented toward the horizontal direction in the maps, induce hot spots (corresponding to the brightest yellow points) around corners of two nearly-touching inclusions (e.g. top-right on each of the fours maps).

Numerical results are compared to available analytical bounds, "Zero-order" (ZO), Wiener (W) and Hashin and Shtrikman's (HS) bounds. Each of these bounds delimit the minimal admissible subdomains of the complex plane where the effective permittivity components stand [19], depending on the available microstructural information. These are respectively: no information beside the permittivity functions of glass and $\mathrm{TiO}_{2}$ is known (ZO); the local permittivity and the phase volume fractions are known (W); the microstructure is, additionally, isotropic (HS). As a consequence, the domain (HS) is enclosed in domain (W) itself enclosed in domain (ZO), and each of these are made of two points (or corners) and delimited by circular arcs. Additionally, (ZO), (W) and (HS) bounds are exact to zero, first and second order in the contrast, in the limit where the contrast of properties is infinitesimal. Additional third or fourth-order bounds rely on more complex microstructural information [10] and are not used in this work. We refer to Chapter 27 of [20] for a review. Although (HS) bounds are valid for isotropic microstructures only, they provide helpful approximations for deposit models which are transversely isotropic but close to isotropic.

\section{Anisotropy of the permittivity tensor}

The full permittivity tensor of deposit models is first computed on microstructures of types (i) and (iii) with wavelength $381.24 \mathrm{~nm}$, i.e. violet color, using full-fields computations.

\subsection{Spheres}

As previously observed for the microstructure in section (2.3), transverse isotropy is recovered with a good approximation. For instance, for a microstructure with spherical inclusions and $16 \%$ volume fraction, the effective permittivity reads, in $F / m$ :

$$
\varepsilon_{\mathrm{S}-16 \%}^{*}=\left(\begin{array}{ccc}
2.834+4.54210^{-3} i & 7.810^{-4}+1.410^{-5} i & -9.810^{-4}-1.910^{-5} i \\
- & 2.837+4.60310^{-3} i & 2.110^{-4}+3.010^{-6} i \\
- & - & 2.856+4.97710^{-3} i
\end{array}\right)
$$


where the component $\varepsilon_{i j}^{*}$ is on line $i$ and column $j$, if $(1,2,3)=(x, y, z)$, and the matrix is completed by symmetry. The tensor is, with a good approximation, a diagonal matrix because the element outside the diagonal are close to zero, accordingly the eigen values are close to the diagonal values of the matrix. On the diagonal, the component $\varepsilon_{z z}^{*}$ is slightly different from $\varepsilon_{x x}^{*} \approx \varepsilon_{y y}^{*}$, as a consequence of the deposit direction. This effect is however small in the case of spheres. Furthermore, the imaginary part is much smaller than the real part. This accounts for a small dispersion and high percolation effect of the glass resin. Moreover, the difference between $\varepsilon_{z z}^{*}$ and other diagonal components is stronger for the imaginary part than it is for the real part. Indeed, using the anisotropy indicator $\nu_{123}(a)=(1 / 2)\left(\left|a_{11}-a_{33}\right| / a_{11}+\left|a_{22}-a_{33}\right| / a_{22}\right)$, it is found that $\nu_{123}\left(\operatorname{Re} \varepsilon^{*}\right)=0.7 \%$ whereas $\nu_{123}\left(\operatorname{Im} \varepsilon^{*}\right)=8.9 \%$. Accordingly, the deposit model transverse isotropy has a much stronger effect on the effective imaginary part.

The same remarks apply to the effective permittivity tensor for a microstructure with spherical inclusions and higher volume fraction $18 \%$ :

$$
\varepsilon_{\mathrm{S}-18 \%}^{*}=\left(\begin{array}{ccc}
2.900+5.25110^{-3} i & 1.510^{-3}+2.810^{-5} i & -7.610^{-4}+1.410^{-5} i \\
- & 2.899+5.23610^{-3} i & -1.810^{-4}+2.710^{-6} i \\
- & - & 2.919+5.63310^{-3} i
\end{array}\right)
$$

where, in this case, it is found that $\nu_{123}\left(\operatorname{Re} \varepsilon^{*}\right)=0.7 \%$ whereas $\nu_{123}\left(\operatorname{Im} \varepsilon^{*}\right)=7.4 \%$. Yet again, the deposition direction has a much stronger effect on the imaginary part than on the real part. This difference however is reduced when the volume fraction increases; this is related to connection effects in the microstructure: when the number of spheres is small, paths passing through connected inclusions tend to be oriented in the deposit direction. On the contrary, when the volume fraction increases, these paths take much more arbitrary directions.

\subsection{Rhombus-shaped inclusions}

The full permittivity tensor of a deposit model of aligned rhombi inclusions with $18 \%$ volume fraction reads:

$$
\varepsilon_{\mathrm{R} \text {-Al. }}^{*}=\left(\begin{array}{ccc}
2.949+6.41810^{-3} i & 4.210^{-4}+8.110^{-6} i & 4.410^{-4}+1.010^{-5} i \\
- & 2.857+4.63510^{-3} i & -2.010^{-4}-3.410^{-6} i \\
- & - & 2.990+7.44310^{-3} i
\end{array}\right)
$$

The material is not transverse isotropic anymore but orthotropic, as a consequence of the inner anisotropy of the inclusions. Rhombi are not isotropic in the $(x, y)$ plane because they are aligned along the same preferential directions. This anisotropy is even stronger than the one introduced by the deposit model. Transverse isotropy is recovered in the rotated rhombi microstructure where

$$
\varepsilon_{\mathrm{R} \text {-Rot. }}^{*}=\left(\begin{array}{ccc}
2.908+5.61510^{-3} i & 1.510^{-2}+2.910^{-4} i & -1.110^{-4}-1.410^{-6} i \\
- & 2.915+5.76410^{-3} i & -9.410^{-4}-2.110^{-5} i \\
- & - & 2.976+7.11110^{-3} i
\end{array}\right)
$$


It is found that $\nu_{123}\left(\operatorname{Re} \varepsilon^{*}\right)=2.2 \%$ whereas $\nu_{123}\left(\operatorname{Im} \varepsilon^{*}\right)=25 \%$. The high values are a consequence of the inclusions anisotropy, and not only of the anisotropy induced by the deposit model.

Hereafter, the diagonal values of the effective permittivity tensor $\varepsilon^{*}$ for the various spherical and rhombus-shaped microstructures are computed at varying wavelengths in the visible spectrum. These bounds delimit domains in the complex plane; for the sake of clarity, such domains are only plotted at the lowest and highest wavelengths, plus the point with the highest effective imaginary value.

\section{Bounds for the effective response}

Cole-cole graphs representing the effective permittivity eigen values at varying wavelengths are shown in Figure 6a, for microstructures with spherical grains. As expected, the numerical results respect the Wiener bounds, represented, for the sake of clarity, at the smallest wavelength only $(\lambda=381 \mathrm{~nm})$. At other wavelengths, the Wiener bounds are not violated either (not shown). It appears that the eigen value transverse to the deposit microstructure, i.e. $\varepsilon_{x x}^{*} \approx \varepsilon_{y y}^{*}$, is very close to one of the two "corners" of Hashin and Shtrikman's domain. The same phenomenon is observed at all wavelengths (not shown). This HS corner corresponds to the lower HS bound if the permittivity were real, and is attainable by Hashin's coated sphere assemblage with glass as the coating sphere.

The very small difference between the HS lower bound and the effective properties of a model of non-interpenetrable spheres has been observed previously for the thermal and elastic properties of fibre composite materials generating by $2 \mathrm{D}$ sections a hard-core structure of discs, when the inclusions conductivity is higher than the embedding matrix [21]. It is in particular valid when there is a minimal distance of repulsion between the spheres, or when spheres only rarely touch. Spheres connexions are indeed not favoured by the deposit model in the $(x, y)$ plane, which explains the numerical results obtained for the transverse permittivity eigen value.

The eigen value parallel to the deposit direction, $\varepsilon_{z z}^{*}$, however, is different from the lower HS bounds, and close to one of the arc circles joining the two corners of HS's domain, precisely the one with the overall lowest imaginary part. Yet again, this phenomenon is observed at all wavelengths (not shown). This effect, although it is probably only an approximate result, is a consequence of the higher level of connexions between touching spheres in the $z$ direction for the deposit model, and of the fact that the spheres have non-zero imaginary part. The fact that the effective permittivity in the parallel direction is close to one of HS's circle arc mimic the behavior of these composites in the real case. It is noted that the 2D HS bounds (not plotted) do not provide any useful approximation of the effective permittivity components, so that the effect of $3 \mathrm{D}$ deposition can not be neglected.

Cole-cole plots for the effective properties eigen values are shown in Figure $6 \mathrm{~b}$ for aligned rhombus-shaped inclusions with volume fraction $18 \%$. The latter are within the Wiener bounds but violate the Hashin and Shtrikman bounds. Indeed, the microstructure is in this case not isotropic. As seen in the plots, the effective properties are close to the "lowest" arc circle of the Wiener bounds. Here again, the percolation paths of the 
embedded glass, much more dense in the deposit models than that of the inclusions, account for this effect. It is noted (see [20]) that points on the lowest Wiener curve are exactly realizable by a model of coated ellipsoids where glass is the coating phase. The latter model mimic the inclusions anisotropy in the present case.

Although the real part of the effective properties of the microstructure differ very little when considering spherical or rhombus-shaped polyhedrons, this is not true for the imaginary part. The latter show noticeable differences with higher values for rhombi. This behavior stands even in the case of transverse isotropy and leads to higher absorption effects at the considered wavelength, and to color differences, as the latter are highly sensitive to very small difference in the imaginary part.

\section{Representative Volume Element}

The representative volume element (RVE) is a concept used to define the volume $V$ of an heterogeneous material that is, under an assumption of ergodicity, sufficiently large to be statistically representative of the composite[12]. As recalled in section 3, the ergodic property of the model is useful to compute the effective properties $\mathbf{E}$ and $\mathbf{D}$ by means of spatial averages over the volume $V$. From the classical geostatistical theory [18] the variance $D_{Z}^{2}(V)$ of the average value $\langle Z\rangle_{V}$ over the volume $V$ is defined for a stationary ergodic random function $Z(x)$ and $D_{Z}^{2}(V) \simeq D_{Z}^{2} \frac{A_{3}}{V}$, when $V$ is large enough, where $D_{Z}^{2}$ is the point variance of $Z(x)$ and $A_{3}$ is the integral range of $Z$. Equaling $Z$ with the displacement field component $D_{x}$, the latter provides a direct relation between error deviations due to finite size effects on the apparent permittivity, and the volume size. More precisely, for $n$ realizations of volumes $V \gg A_{3}$, the absolute error on the effective property is $\eta_{\mathrm{abs}}=\frac{2 D_{Z}(V)}{\sqrt{n}}$ and the relative error is $\eta_{\mathrm{rel}}=\eta_{\mathrm{abs}} / Z$. Accordingly, when considering one realization $(n=1)$, as in this work, the RVE size $V$ depends on the error $\eta_{\text {rel }}$ by

$$
\eta_{r e l}(V) \simeq 2 \sqrt{\frac{D_{Z}^{2} A_{3}}{V\langle Z\rangle}}, \quad V \gg A_{3} .
$$

The ergodic property of the microstructure was used to compute $D_{Z}^{2}(V)$ over different (independent) subvolumes of increasing size from $30^{3}$ to $300^{3}$ or $600^{3}$ voxels, within deposit models with $600^{3}$ and $1200^{3}$ voxels image.

The variances of the volume fraction $D_{Q}^{2}(V)$ and of the real and imaginary part of the displacement field $D_{\operatorname{Re}\left(\mathbf{D}_{x}\right)}^{2}(V)$ and $D_{\operatorname{Im}\left(\mathbf{D}_{x}\right)}^{2}(V)$ are plotted as a function of $V$ in $\log$ - $\log$ scale in figure 8. The microstructures contains about 1600 spheres, with uniform radius distribution between 20 and $40 \mathrm{~nm}$. The spheres volume fraction is $16 \%$ and the discretization is $1 \mathrm{voxel} / \mathrm{nm}$. The number of voxels is $1200^{3}$. It is noted that the $600^{3}$ microstructures is not representative enough to estimate $A_{3}$. As shown in Figure 8, indeed, the variances exhibit a scaling law $\sim 1 / V^{\nu}$ with $\nu \approx 0.5$ or 0.7 for the displacement fields and microstructure resp., in an intermediate regime when $V<(600 \mathrm{~nm})^{3}$. This particular behavior is a consequence of boundary effects. At the top of the microstructure, corresponding to planes with the highest $z$ coordinate, the deposit model indeed contains much less inclusions, as only spheres that are contained within the boundaries are included 
at the end of the deposit process. Scaling with non-integer exponents for the variance have been found previously in degenerate microstructures exhibiting infinite range correlations, such as fiber media (see $[1,9]$ ).

The variance $D_{\operatorname{Im}\left(\mathbf{D}_{x}\right)}^{2}(V)$ for the imaginary part of the displacement field is much higher than for its real part $D_{\operatorname{Re}\left(\mathbf{D}_{x}\right)}^{2}(V)$. This effect is to be compared with the field maps 7, where the electric displacement field exhibits hot spots. Such hot spots are located in-between two spheres that touch each other or are very close to each other along the direction of the applied macroscopic field, which in this case in the $x$ direction (leftright on the image). This is reminiscent of the thermal response of granular materials as described in [25]. In the deposit models, hot spots influence the average field over subdomains, and according to the map, their effect must be stronger on the imaginary part of the displacement field, where they are much more contrasted. They are also very few and are far from each other, as seen in the top-left map of Figure 7, where only two of them are visible, and therefore induce a high spatial heterogeneity.

The $1200^{3}$ voxels microstructure is large enough to observe the expansion predicted in equation 3. For the microstructure, the point variance and integral range is found to be equal to

$$
D_{Q}^{2}=0.135, \quad A_{3}(Q)=(6.38 \mathrm{~nm})^{3} .
$$

For the displacement field, a numerical fit on the FFT data provides

$$
\begin{aligned}
& A_{3}\left(\operatorname{Re}\left(\mathbf{D}_{x}\right)\right)=3.5510^{5} \approx(71 \mathrm{~nm})^{3} \\
& A_{3}\left(\operatorname{Im}\left(\mathbf{D}_{x}\right)\right)=4.1610^{5} \approx(75 \mathrm{~nm})^{3}
\end{aligned}
$$

It is checked a posteriori that the expansion 3 holds when $V \gtrsim A_{3}$. Furthermore, the relative errors computed for the first component of the electric displacement $\mathbf{D}_{x}$ and for the volume fraction are:

$$
\eta_{\text {rel }}\left(\operatorname{Re}\left(\mathbf{D}_{x}\right)\right)=0.82 \%, \quad \eta_{\text {rel }}\left(\operatorname{Im}\left(\mathbf{D}_{x}\right)\right)=8.84 \%, \quad \eta_{\text {rel }}(Q)=0.07 \%
$$

Alternatively, to achieve an accuracy of $1 \%$ the following minimal volume sizes (in voxels or $\mathrm{nm}^{3}$ ) should be used:

$$
V_{\operatorname{Re}\left(\mathbf{D}_{x}\right)}=1050^{3}, \quad v_{\operatorname{Im}\left(\mathbf{D}_{x}\right)}=5130^{3}, \quad v_{Q}=204^{3},
$$

The hot spots where the displacement field has a local maximum induce size effects which are much bigger than the inclusion size: in the $1200^{3}\left(\mathrm{~nm}^{3}\right)$ simulation the size effects are visible up to cubes of $100^{3}\left(\mathrm{~nm}^{3}\right)$ volumes whereas the pigment volume is of the order of $60^{3}\left(\mathrm{~nm}^{3}\right)$. Such effects are obviously invisible to the human eyes, but pigment with larger (micrometer) size could induce similar phenomena.

\section{Conclusion}

This work focuses on the representation, at the micro and mesoscopic level, of a paint layer, and of its optical response, in the simpler quasi-static hypothesis. A general transverse 
isotropic deposit model suitable to paint layers has been developed, that is generic and practical to implement. Expansion of this model should be studied to increase the pigments volume fractions, e.g. by adequately choosing the position of the pigments before the latter "fall" in the microstructure.

Full-field FFT computations have been undertaken on the various deposit models. The resulting properties are, as expected, transversely isotropic or orthotropic. For the deposit microstructure with spherical pigments, the effective permittivity is very well approximated by the (complex equivalent of) the lower Hashin-Shtrikman bounds in the direction transverse to the deposition. In the direction parallel to it, the effective permittivity lies along the "lower" border of the Hashin-Shtrikman complex domain.

The presence of high local field fluctuations necessitates the use of very large microstructure realizations, that are able to simulate a representative volume element (RVE, typically $1000 \mathrm{~nm}^{3}$ in the present case). The RVE size is much larger than the pigment size, in particular for the imaginary part of the displacement field, which generates the color at the macroscopic scale. Stronger effects are expected in real paint materials where the pigments tend to aggregate with each other and form clusters, requiring multiscale microstructure models, such as already made in [7].

Acknowledgments: This work was supported by a grant from ANR (reference: 2011 RMNP 014). The authors are grateful to Mona Ben Achour, Anthony Chesnaud, Alain Thorel and Fernando da Graça for useful discussions and information during this study.

\section{References}

[1] H. Altendorf, D. Jeulin, and F. Willot. Effect of the random distribution of fibers on the effective properties: application to glassfiber- reinforced composites. Composites Part A, submitted.

[2] H. Du, H. Chen, B. K. Moom, J. H. Shin, and S. W. Lee. Effect of plasma spraying condition on deposition efficiency, microstructure and microhardness of tio2 coating. AZo J. of Materials Online, (1), 2005.

[3] D. J. Eyre and G. W. Milton. A fast numerical scheme for computing the response of composites using grid refinement. The Eur. Phys. J. Appl. Phys., 6(1):41-47, 1999.

[4] M. Gotić, G. Dražić, and S. Musić. Hydrothermal synthesis of $\alpha-\mathrm{Fe}_{2} \mathrm{O}_{3}$ nanorings with the help of divalent metal cations, $\mathrm{Mn}^{2+}, \mathrm{Cu}^{2+}, \mathrm{Zn}^{2+}$ and $\mathrm{Ni}^{2+}$. J. of Molecular Structure, (993), 2011.

[5] X. Huang, J. Guan, Z. Xiao, G. Tong, F. Mou, and X. Fan. Flower-like porous hematite nanoarchitectures achieved by complexation-mediated oxidation-hydrolysis reaction. J. of Colloid and Interface Sc., (357), 2011.

[6] M. I. Idiart, F. Willot, Y.-P. Pellegrini, and P. Ponte Castañeda. Infinite-contrast periodic composites with strongly nonlinear behavior: Effective-medium theory versus full-field simulations. Int. J. of Sol. and Struct., (46), 2009. 
[7] A. Jean, F. Willot, S. Cantournet, S. Forest and D. Jeulin. Large-Scale computations of effective elastic properties of rubber with carbon black fillers. International Journal for Multiscale Computational Engineering, (9), 2011.

[8] D. Jeulin. Random structures in physics. In Michel Bilodeau, Fernand Meyer, and Michel Schmitt, editors, Space, Structure and Randomness, volume 183 of Lecture Notes in Statistics, pages 183-219. Springer New York, 2005.

[9] D. Jeulin. Variance scaling of boolean random varieties. http://hal.archivesouvertes.fr/hal-00618967, 2011.

[10] D. Jeulin and L. Savary. Effective complex permittivity of random composites. $J$. Phys. I France, 7(9):1123-1142, 1997.

[11] S. K. Kanaun and D. Jeulin. Effective field method in the problem of electromagnetic wave propagation through a medium with isolated inclusions. J. of Electromagnetic Waves and Appl., 11(11):1531-1566, 1997.

[12] T. Kanit, S. Forest, I. Galliet, V. Mounoury, and D. Jeulin. Determination of the size of the representative volume element for random composites: statistical and numerical approach. Int. J. of Sol. and Struct., 40(13-14):3647-3679, 2003.

[13] L. D. Landau and E. M. Lifshitz. Course of Theoretical Physics: Vol.: 8: Electrodynamics of Continuous Media, chapter XI. Pergamon Press, 1960.

[14] S. Lian, H. Li, X. He, Z. Kang, Y. Liu, and S. T. Lee. Hematite homogeneous core/shell hierarchical spheres: surfactant-free solvothermal preparation and their improved catalytic property of selective oxidation. J. of State Chemistry, (185), 2012.

[15] J. Lu, D. Chen, and X. Jiao. Fabrication, characterization and formation mechanism of hollow spindle-like hematite via solvothermal process. J. of Colloid and Interface Sc., (303), 2006.

[16] S. Manickavasagam, C. Saltiel, and H. Giesche. Characterization of colloidal hematite particle shape and dispersion behavior. J. of Colloid and Interface, (280), 2004.

[17] G. Matheron. Eléments pour une théorie des milieux poreux. Masson, Paris, 1967.

[18] G. Matheron. The theory of regionalized variables and its applications. Les Cahiers du Centre de morphologie mathématique de Fontainebleau. École nationale supérieure des mines, Paris, 1971.

[19] G. W. Milton. Bounds on the complex dielectric constant of a composite material. Appl. Phys. Let., 37(3):300-302, 1980.

[20] G. W. Milton. The theory of composites. Cambridge monographs on applied and computational mathematics. Cambridge University Press, Cambridge, 2002. 
[21] M. Oumarou, D. Jeulin, and J. Renard. Multi-scale statistical approach of the elastic and thermal behavior of a thermoplastic polyamid-glass fiber composite. Technische Mechanik, (32):484-506, 2012.

[22] G.-S. Park, D. Shindo, Y. Waseda, and T. Sugimoto. Internal structure analysis of monodispersed pseudocubic hematite particles by electron microscopy. J. of Colloid and Interface Sc., (177), 1996.

[23] S. Y. Reyes López, J. Serrato Rodríguez, and S. Sugita Sueyoshi. Low-temperature formation of alpha alumina powders via metal organic synthesis. AZo J. of Materials Online, (2), 2006.

[24] T. E. Tiwalda and M. Schubertb. Measurement of rutile $T_{i} O_{2}$ dielectric tensor from 0.148 to $33 \mu \mathrm{m}$ using generalized ellipsometry. In Optical Diagnostic Methods for Inorganic Materials II, Leonard M. Hanssen, Editor, Proc. of SPIE, 2000.

[25] F. Willot, L. Gillibert, and D. Jeulin. Microstructure-induced hotspots in the thermal and elastic responses of granular media. Int. J. of Solids and Struct., (10), 2013.

[26] F. Willot and D. Jeulin. Elastic and electrical behavior of some random multiscale highly-contrasted composites. Int. J. of Multiscale Comp. Eng., 9(3):305-326, 2010.

[27] F. Willot, Y.-P. Pellegrini, I. Idiart, M., and P. Ponte Castañeda. Effective-medium theory for infinite-contrast two-dimensionally periodic linear composites with strongly anisotropic matrix behavior: Dilute limit and crossover behavior. Phys. Rev. B, (104111), 2008.

[28] Y. Xu, S. Yang, G. Zhang, Y. Sun, D. Gao, and Y. Sun. Uniform hematite $\alpha-$ $\mathrm{Fe}_{2} \mathrm{O}_{3}$ nanoparticles: morphology, size-controlled hydrothermal synthesis and formation mechanism. Materials Letters, (65), 2011.

[29] G.-Y. Zhang, Y. Feng, Xu Y.-Y., D.-Z. Gao, and Y.-Q. Sun. Controlled synthesis of mesoporous $\alpha-\mathrm{Fe}_{2} \mathrm{O}_{3}$ nanorods and visible light photocatalytic property. Materials Res. Bulletin, (47), 2012. 

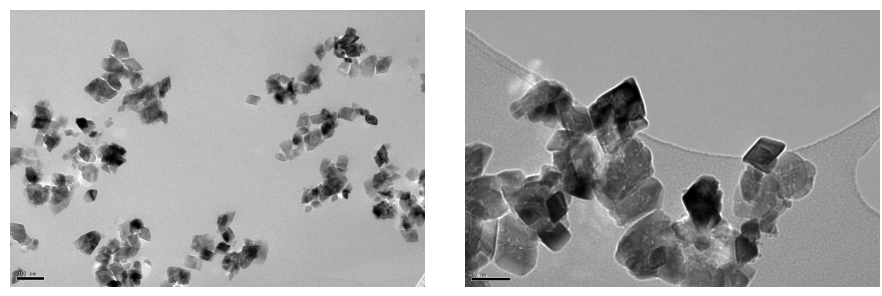

Figure 1: SEM micrograph of $\mathrm{Fe}_{2} \mathrm{O}_{3}$ powders (c) 2012 Mona Ben-Achour, PhD thesis, Centre des Matériaux, Mines ParisTech.
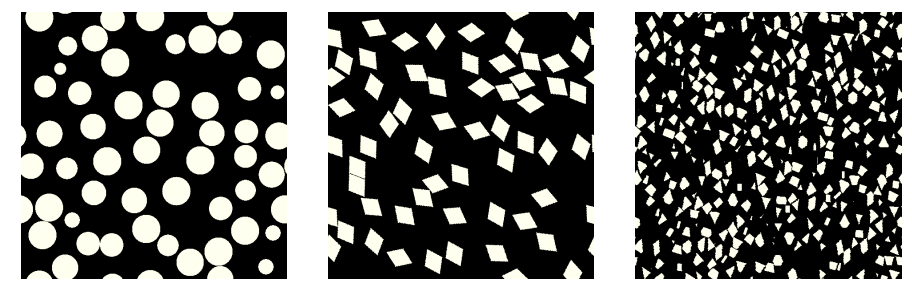

Figure 2: Cut along the middle plane $z \equiv \bar{z} / 2$ of deposit microstructures with spherical inclusions of fixed radius (left), randomly rotated rhombi in the $(x, y)$ plane (middle) and randomly rotated rhombi in the $(x, y)$ and $(y, z)$ plane (right). The microstructure resolutions are $600^{3}$ voxels or $\mathrm{nm}^{3}$. 


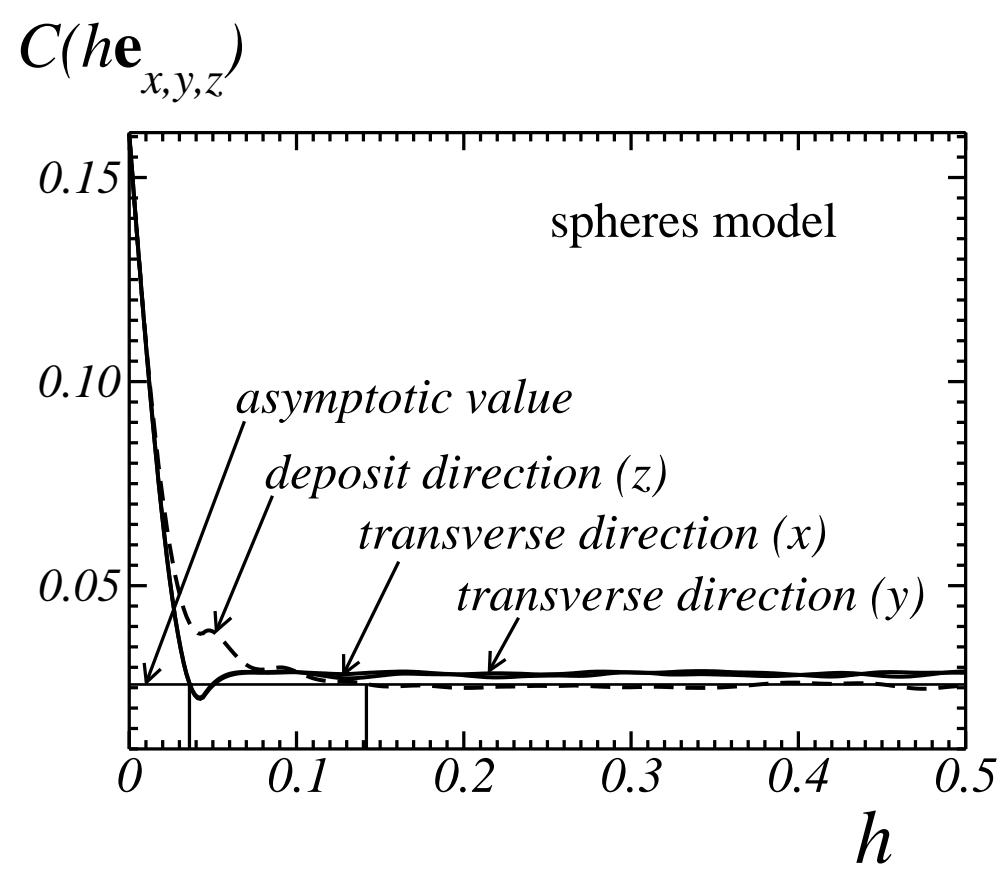

Figure 3: Covariance $C_{Q}\left(h \mathbf{e}_{x, y, z}\right)$ in the $x, y$ and $z$ directions (transverse and parallel to the deposit direction, dotted and solid lines, resp.) of a deposit microstructure with spheres of equal radius. The value $h$ is normalized by the microstructure size. The covariances are equal to the volume fraction of spheres $f=16 \%$ at $h=0$ whereas they tend to the asymptotic value $f^{2} \approx 0.025$ at long distances (thin horizontal line). 


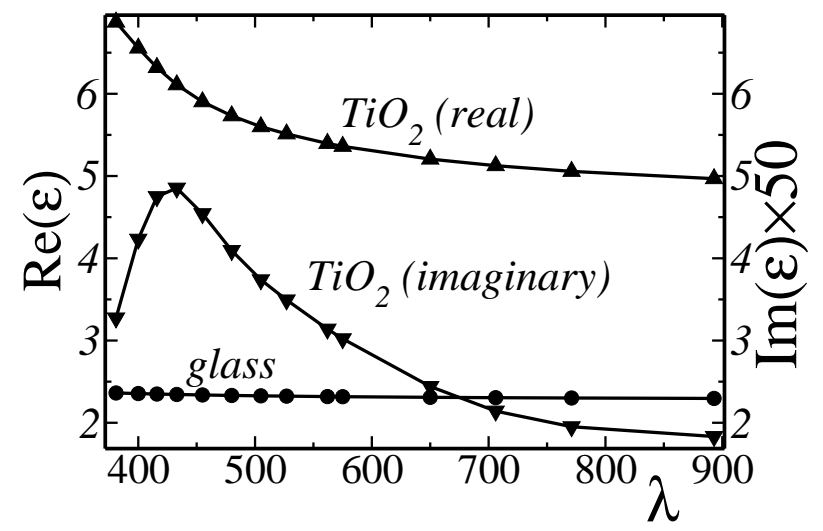

Figure 4: Permittivity functions $\varepsilon$ of glass (dots) and $\mathrm{TiO}_{2}$ (up and down triangles) in Farads per meter, as a function of wavelength $\lambda$ in the visible spectrum, in nanometers. The imaginary part of the permittivity of glass is 0 . That of $\mathrm{TiO}_{2}$ was multiplied by 50 for comparison on the graph. 

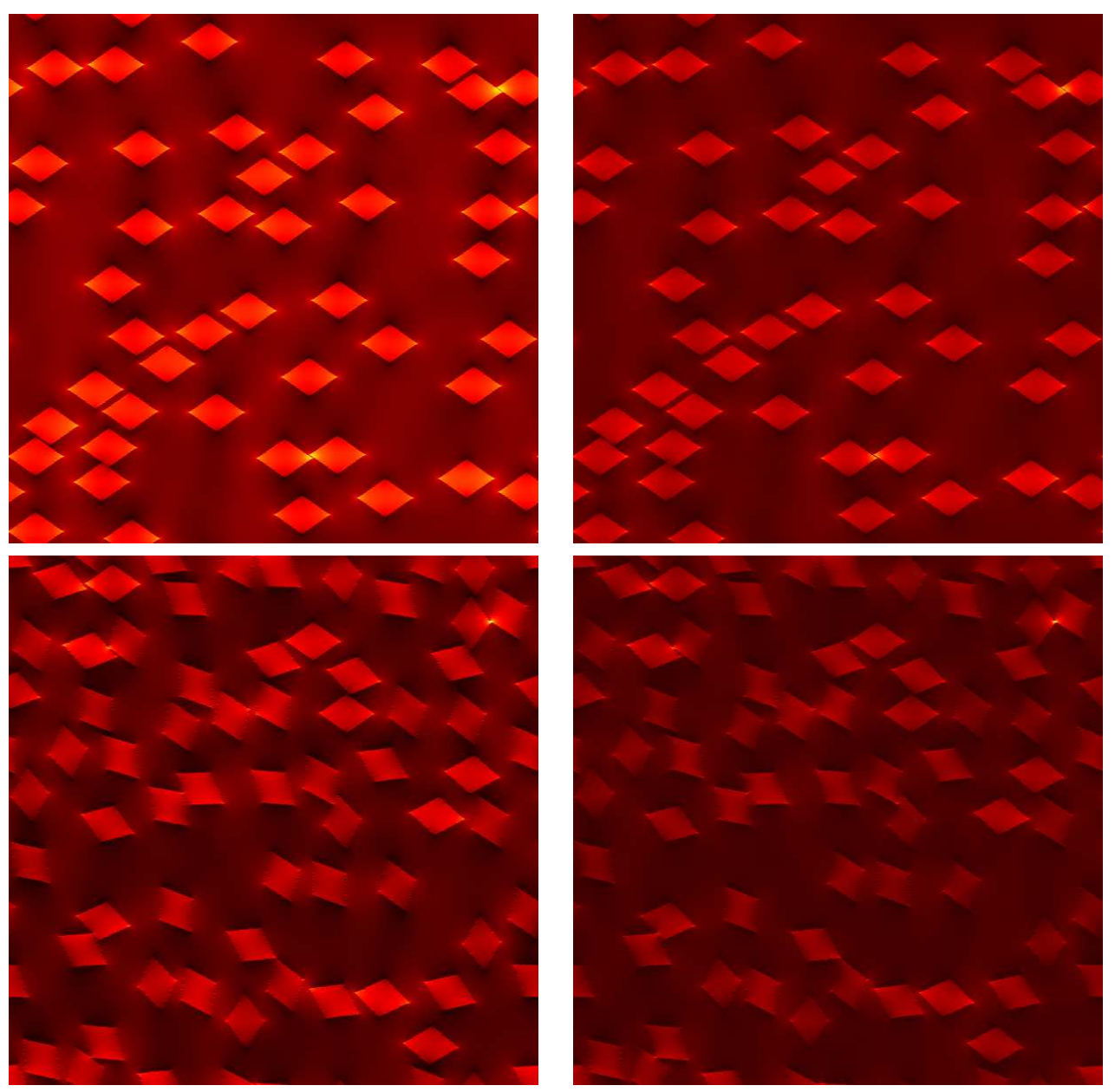

Figure 5: 2D cut along the middle plane $z \equiv \bar{z} / 2$ of the real (left) and imaginary part (right) of the $x$-component of the displacement field $D_{x}$, for the rhombus-shaped deposit microstructure with aligned and non-aligned inclusions, with $600^{3}$ (top) and $1200^{3}$ (bottom) voxels discretization. A macroscopic electric field $\langle\mathbf{E}\rangle_{Q}=\mathbf{E}_{0}=\mathbf{e}_{x}$ is imposed in the $x$ direction. 


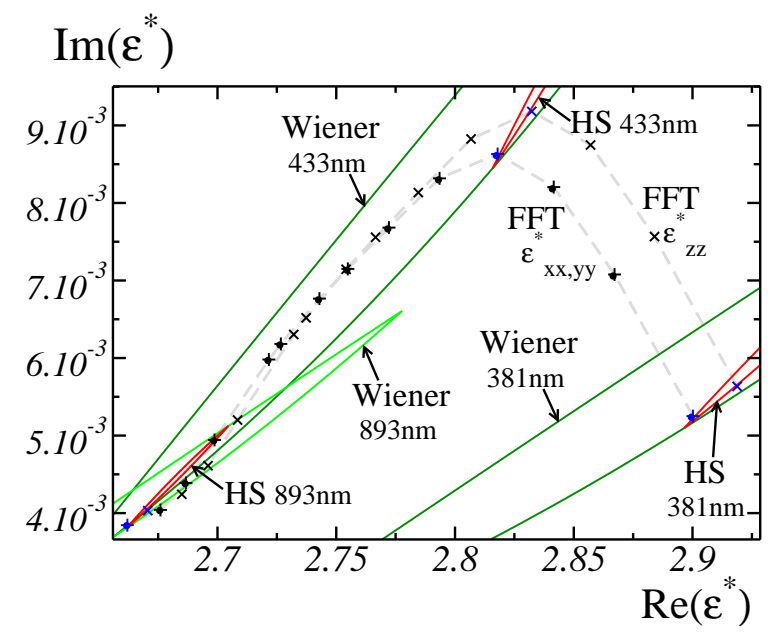

(a)

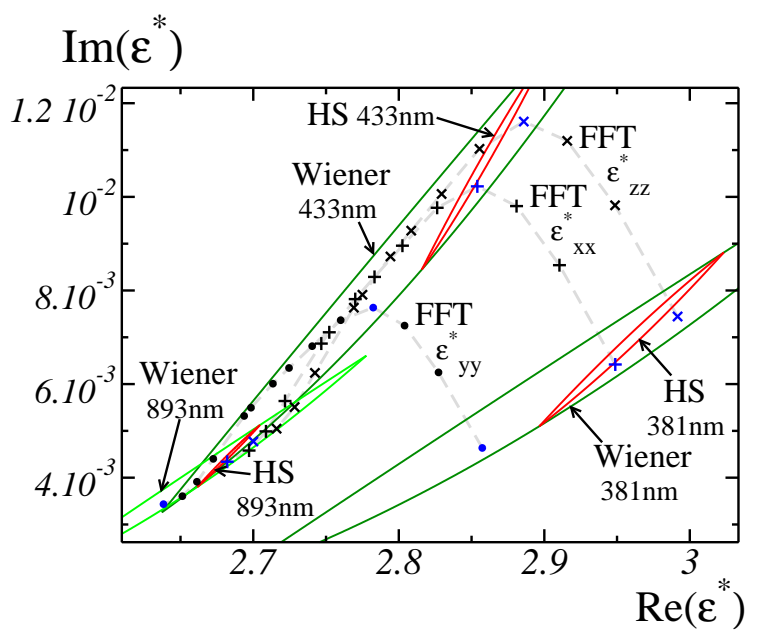

(b)

Figure 6: Cole-cole plots of the effective permittivity components $\varepsilon_{x x, y y, z z}^{*}$ (symbols + , $\bullet$ and $\times$ resp., joined by dotted gray lines), as computed by FFT, for a deposit microstructure made of spheres (a) or aligned rhombi inclusions (b) with volume fraction $18 \%$. The wavelength takes the values $\lambda=381,400,416,433,455,480,505,527,562,575,650,706$, 771 , and 893nm (right to left). Domains delimited by the Wiener and Hashin-Shtrikman's bounds are shown in green and red, resp., for the values $\omega=381,893$ and $433 \mathrm{~nm}$ with corresponding FFT results shown in blue. 

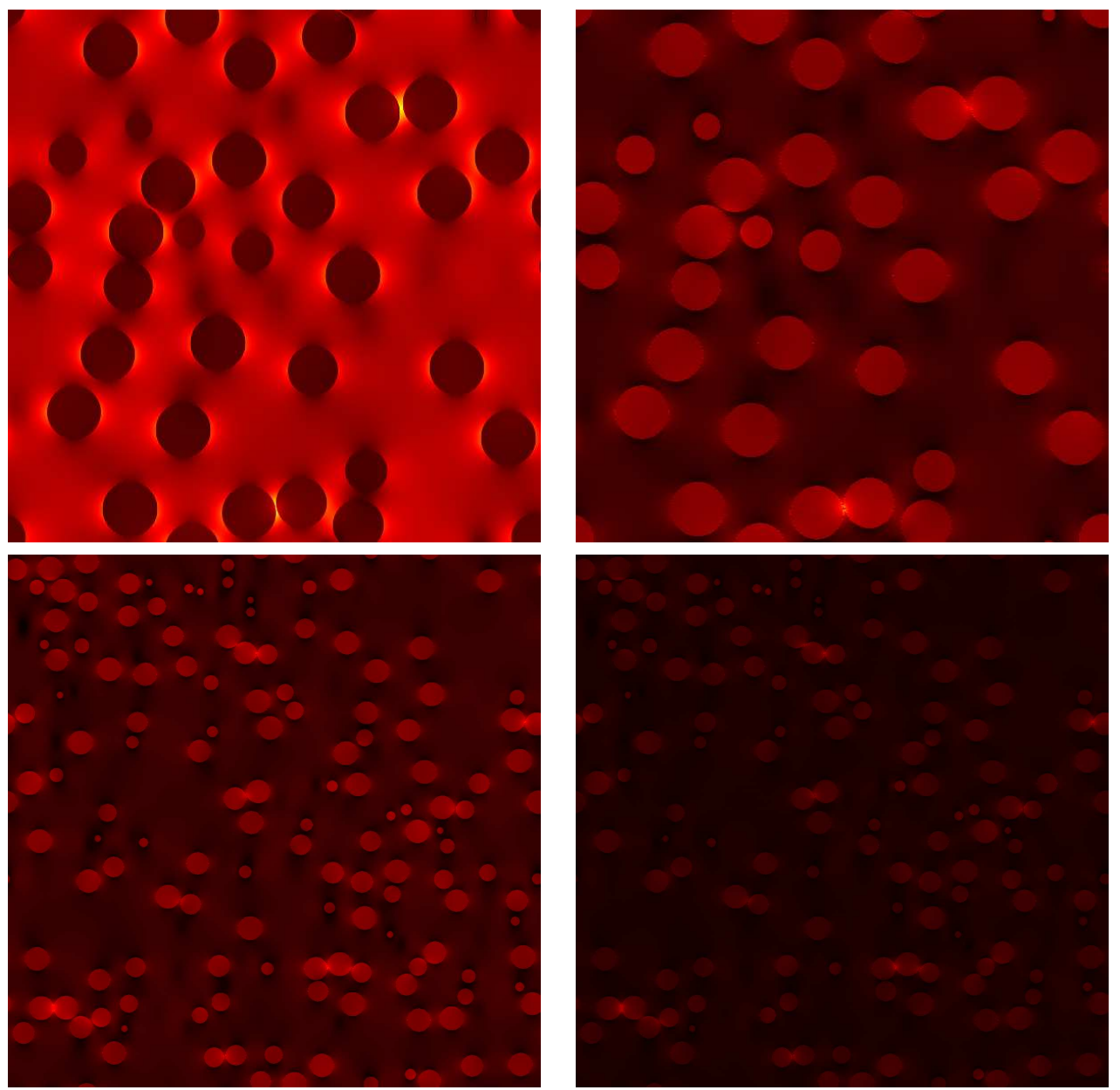

Figure 7: 2D cut along the middle plane $z \equiv \bar{z} / 2$ of the real (left) and imaginary part (right) of the $x$-component of the displacement field $D_{x}$, for the spherical deposit microstructure (ii). The 3D microstructures in the top (resp. bottom) maps contain 405 (resp. 7529) spheres with uniform radius distribution between $20 \mathrm{~nm}$ and $40 \mathrm{~nm}$. The discretization employed is $600^{3}$ voxels or $\mathrm{nm}^{3}$ (top and bottom, resp.). The spheres volume fraction is about $16 \%$ and a macroscopic electric field $\langle\mathbf{E}\rangle_{Q}=\mathbf{E}_{0}=\mathbf{e}_{x}$ is applied in the $x$ direction. 


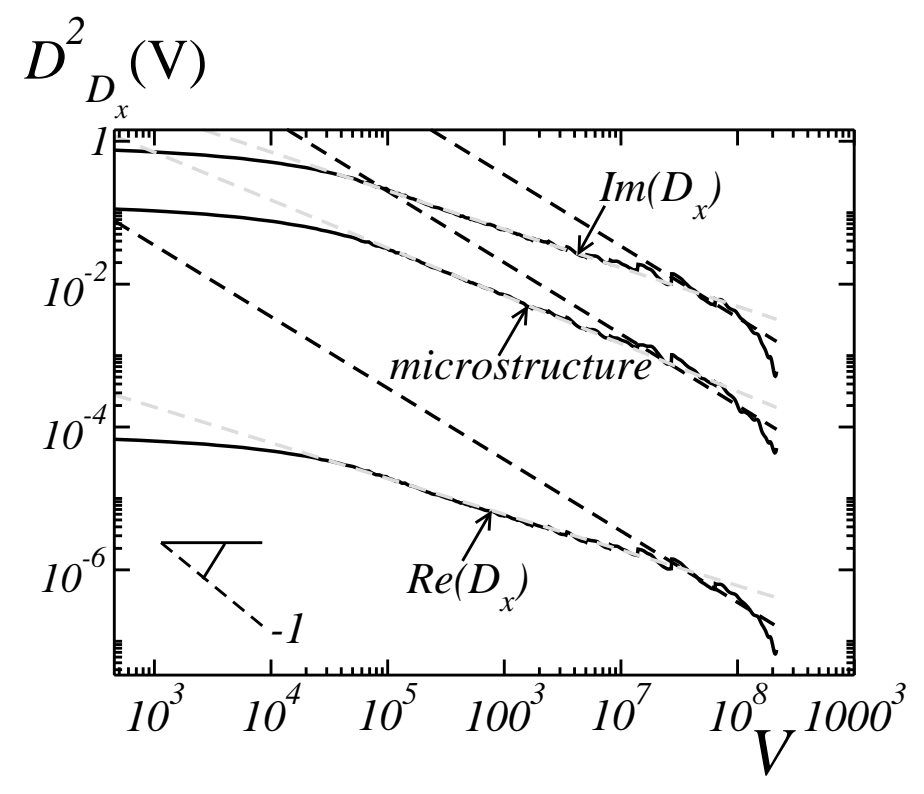

Figure 8: Variance $D_{D_{x}}^{2}(V)$ of the mean of the real and imaginary parts of the electric displacement component $D_{x}$ over $V$, and of the microstructure volume fraction $D_{Q}^{2}(V)$ as a function of the subvolume size $V$ in a deposit model of spheres with $1200^{3}$ voxels. These quantities are equivalent to the apparent permittivity $\varepsilon_{x x}$ and to the volume fraction of spheres, respectively (a macroscopic electric field $\left\langle E_{x}\right\rangle=E_{x}^{0}$ is applied). The asymptotic expansion $D_{D_{x}, Q}^{2}(V) \sim 1 / V$ (with different prefactors) is recovered when the subvolume $V$ is larger than $600 \mathrm{~nm}^{3}$. At smaller sizes, the variance exhibits a slower decrease, as $D_{D_{x}}^{2} \sim 1 / V^{0.5}$ and $D_{Q}^{2} \sim 1 / V^{0.67}$ for the displacement field and microstructure respectively (dotted gray lines). 